\title{
Selective Behavioral and Neurochemical Effects of Cholinergic Lesions Produced by Intrabasalis Infusions of 192 IgG-Saporin on Attentional Performance in a Five-Choice Serial Reaction Time Task
}

\author{
J. McGaughy, J. W. Dalley, C. H. Morrison, B. J. Everitt, and T. W. Robbins \\ Department of Experimental Psychology, University of Cambridge, Cambridge CB2 3EB, United Kingdom
}

The effects of the cholinergic immunotoxin $192 \mathrm{lgG}$-saporin (SAP) $(0.0,0.15$, or $0.45 \mu \mathrm{g} / \mu \mathrm{l} ; 0.5 \mu \mathrm{l} /$ hemisphere) infused into the area of the nucleus basalis magnocellularis (NBM) of rats were tested in a five-choice serial reaction time task (5CSRTT) designed to assess visual attention. The effects of this manipulation on acetylcholine efflux in the medial frontal cortex were determined using in vivo microdialysis during the 5CSRTT. Rats with extensive lesions of the NBM (SAP HIGH) showed an array of behavioral deficits in the 5CSRTT hypothesized to represent deficits in central executive function that were associated with severe deficits in accuracy. Lengthening the stimulus duration ameliorated these deficits. Rats with restricted lesions of the NBM (SAP LOW) showed impairments over time on task when tested under standard conditions that were exacerbated by increases in the event rate. The number of choline acetyl-

Converging evidence from several sources supports an important role for the basal forebrain cholinergic system in aspects of arousal and attentional function. Stimulation of the nucleus basalis magnocellularis (NBM) increases processing of behaviorally relevant stimuli (Metherate and Weinberger, 1990; Metherate and Ashe, 1993), and lesions of this area cause slowing of cortical EEG activity (Detari et al., 1999; Detari, 2000). Lesions of the NBM also impair various attentional functions, including a version of Posner's covert orienting test of visuospatial attention for primates (Voytko et al., 1994; Voytko, 1996), a five-choice serial reaction time task (5CSRTT) (Robbins et al., 1989; Muir et al., 1994) and other tests that assess "incremental" (Chiba et al., 1995), divided (Turchi and Sarter, 1997), or sustained (McGaughy et al., 1996) attention in rats. Recent data have reported increases in acetylcholine (ACh) efflux in the medial prefrontal cortex (mPFC) of rats performing both the 5CSRTT (Passetti et al., 2000) and a sustained attentional task (Himmelheber et al., 2000). However, many of the previous NBM lesion studies are potentially contaminated by the lack of cholinergic selectivity of the lesioning agent. For example, Muir et al. (1994) used the excitotoxin AMPA to demonstrate impairments in the 5CSRTT,

\footnotetext{
Received May 29, 2001; revised Oct. 18, 2001; accepted Nov. 15, 2001.

This research was supported by a Wellcome Trust Program grant and completed within a Medical Research Council Cooperative in Brain, Behavior and Neuropsychiatry. J.M. was supported by a fellowship from the Human Frontiers Science Program Organization (LT-0025-1998B). We thank Helen Sweet and David Theobald for histological and technical assistance.

Correspondence should be addressed to Dr. Jill McGaughy, Boston University, Department of Psychology, 64 Cummington Street, Boston, MA 02215. E-mail: drjillmc@bu.edu.

Copyright (C) 2002 Society for Neuroscience $\quad 0270-6474 / 02 / 221905-09 \$ 15.00 / 0$
}

transferase-immunoreactive cells in the area of the NBM but not the vertical limb of the diagonal band correlated significantly with accuracy in the task. SAP HIGH rats had significantly lower levels of cortical acetylcholine (ACh) efflux relative to SHAM both before and during the 5CSRTT. SAP LOW rats showed significantly higher levels of cortical ACh efflux before but not during the 5CSRTT. Cortical ACh efflux increased in all rats with the onset of the attentional task. These data provide the first direct evidence for a relationship between selective damage in the basal forebrain with decreased cortical ACh efflux and impaired attentional function.

Key words: 192 IgG-saporin; attention; in vivo microdialysis; prefrontal cortex; nucleus basalis magnocellularis; basal forebrain and Voytko et al. (1994) used ibotenic acid. Furthermore, no data are available regarding the simultaneous assessment of the effects of NBM lesions on attentional performance and cortical ACh efflux.

Because there is now a large body of data regarding the underlying neural substrates of the 5CSRTT (Muir et al., 1996a; Baunez and Robbins, 1997) and their neuromodulation by ascending neurotransmitter systems (Carli et al., 1983; Robbins and Everitt, 1995), it would be informative to examine the precise role of cholinergic mechanisms within the mPFC during this task and the causal status of increased ACh efflux observed in freely moving animals. The current study used multiple doses of 192 IgG-saporin (SAP) infused into the NBM to produce differential levels of cortical cholinergic denervation. The 5CSRTT has been modified to emphasize aspects of sustained attention, including the effects of time on task and manipulations of event rate, because this type of attention has been found to be sensitive to intra-NBM immunotoxic lesions (McGaughy et al., 1996). The present study also assessed changes in task parameters, including changes in temporal predictability and stimulus duration similar to those used previously (Muir et al., 1994, 1995, 1996b) to allow comparisons of the current findings with those produced with excitotoxins. The effects of NBM lesions on cortical ACh and noradrenaline (NA) efflux were assessed using in vivo microdialysis during task performance because both neurotransmitters have been implicated in mediating sustained attentional performance (Aston-Jones et al., 1994, 2000; Everitt and Robbins, 1997). Although other studies assessed the attentional (Waite et al., 1999) and neurochemical effects of SAP lesions (Fadel et al., 
1996), this is the first to combine these methods to determine how the loss of cholinergic cell bodies in the NBM influences cortical ACh efflux and attention.

\section{MATERIALS AND METHODS}

\section{Experiment 1}

Twenty Lister hooded, male rats (Harlan, Olac, Bicester, UK) began behavioral training. Two of the lesioned animals failed to maintain sufficient post-surgical body weight and were killed. Four rats damaged their microdialysis probes and so were excluded from the study. Rats were housed in pairs until after surgery. Cages were maintained in a temperature- and humidity-controlled room with a $12 \mathrm{hr}$ light/dark cycle (on 8:30 P.M.). Water was available ad libitum, but food was restricted to $18 \mathrm{gm} / \mathrm{d}$. All procedures were conducted in accordance with the U. K. Animals (Scientific Procedures) Act 1986.

Apparatus. Rats were tested using a system of eight, nine-hole testing chambers described in detail elsewhere (Carli et al., 1983). Each testing chamber was $25 \times 25 \times 25 \mathrm{~cm}$ made of aluminum and equipped with a Plexiglas panel that allowed access to a magazine into which $45 \mathrm{mg}$ of sucrose pellets (Noyes, Sandown Scientific, Middlesex, UK) could be dispensed. A $3 \mathrm{~W}$ bulb illuminated the chambers. Nine apertures were on the rear wall. Four of these were occluded with metal plates, leaving the first, third, fifth, seventh, and ninth holes available to register nose pokes via infrared photocell beams. At the rear of each of these apertures was a $3 \mathrm{~W}$ bulb. Each chamber was situated in a larger, ventilated, soundattenuating chamber. All behavioral measures were recorded using an Acorn Archimedes computer (Cambridge, UK) in conjunction with Arachnid software.

Behavioral training. Rats were trained to nose poke in the aperture where a brief, spatially unpredictable visual stimulus was presented in a 5CSRTT. Initially animals were given extremely long stimuli (e.g., 60 sec) and allowed to nose poke in the presence of the stimulus. If the rat correctly responded to $>80 \%$ of these stimuli for 2 consecutive days, the stimulus duration was reduced until animals were able to respond correctly to $>80 \%$ of 100 trials when presented with a $500 \mathrm{msec}$ stimulus. Animals could make a correct response, e.g., nose poke in the aperture where the stimulus occurred, could make an incorrect response, e.g., nose poke in an aperture where no stimulus was presented, or could fail to respond (an omission). Furthermore, animals could respond before the expiration of the $5 \mathrm{sec}$ intertrial interval (ITI), a premature response, or respond more than once after a correct response (perseverative response). To facilitate acquisition of this task, the animals were given a time out period (houselight off and $5 \mathrm{sec}$ reinforcement suspension) for failing to respond or for responding incorrectly or prematurely. After each trial or time out, rats had to push the magazine panel to initiate the subsequent trial. After animals reached criterion performance with stimuli duration (SD) of $500 \mathrm{msec}$, the contingencies were modified. After a correct trial, rats still had to collect the food pellet from the magazine to initiate the next trial. However, when an incorrect or no response was made, the ITI was immediately initiated either after the response or after the expiration of the $5 \mathrm{sec}$ limited hold period. Furthermore, the houselight remained illuminated during the time out period so that the continuity of the session was not disrupted by the instatement of a dark period. This continuous performance without specific time outs has been shown to increase attentional demands in tests of sustained attention in humans (Parasuraman et al., 1987) and was used to emphasize this dimension of the task. After animals reached $>80 \%$ accuracy and $<25 \%$ omissions, they were considered eligible for surgery.

Experimental design. Rats were randomly assigned to either the vehicle group or the SAP group (Chemicon, Temecula, CA; $0.45 \mu \mathrm{g} / \mu \mathrm{l}$; SAP HIGH). After lesion surgery, rats were given $2 \mathrm{~d}$ of postsurgical recovery and then allowed at least $10 \mathrm{~d}$ of postsurgical retraining in the standard version of the task. After animals' performance stabilized, e.g., $3 \mathrm{~d}$ with levels of percentage correct and omissions differing no more than $15 \%$, rats began a series of behavioral challenges designed to vary the attentional demands of the task. After completion of this series of behavioral challenges presented in a counterbalanced sequence, the rats were implanted with cortical microdialysis probes. Data were collected for 2 in vivo microdialysis days with stimulus durations of 1000 and $500 \mathrm{msec}$, respectively, on days 1 and 2 . The longer stimulus duration on the first day was used to decrease the rate of omissions during this initial in vivo microdialysis testing session.

Postsurgical behavioral testing. Dependent measures recorded during behavioral training included accuracy (correct responses/correct + in- correct responses), percentage of trials omitted, premature responses, perseverative responses, correct response latencies, and magazine latencies. Because SAP HIGH rats showed large impairments in performance, a test day consisting of lengthened SD $(5000 \mathrm{msec}$; ITI $5 \mathrm{sec})$ was implemented. This was hypothesized to decrease attentional demands and facilitate the performance of the rats.

Microdialysis procedures. On a day that in vivo microdialysis was conducted, rats were connected to perfusion lines and placed in the darkened test chamber for $1 \mathrm{hr}$. Basal samples were collected $60 \mathrm{~min}$ later, every $10 \mathrm{~min}$. The task was then initiated with the houselight being illuminated, and performance was measured over $60 \mathrm{~min}$. On completion of the session, animals were returned to their home cages. In vivo microdialysis was conducted over two consecutive daily sessions [for details see Dalley et al. (2001)], each differing only in the length of the stimulus presented (1000 and $500 \mathrm{msec}$ on days 1 and 2, respectively).

Surgical procedures. Detailed information regarding lesioning procedures are available elsewhere (McGaughy et al., 1996). Rats were administered ketamine (Ketaset, Vet Drug, Bury St. Edmunds, UK; $90 \mathrm{mg} / \mathrm{kg}$, i.p.) in combination with xylazine (Rompun, Vet Drug; $6.7 \mathrm{mg} / \mathrm{kg}$, i.p.) to induce anesthesia before being placed in a stereotaxic frame (Kopf, Tijunga, CA). All lesion surgeries were performed using the following coordinates from Paxinos and Watson (1986): incisor bar -3.3, anteroposterior (AP) bregma -0.5 , mediolateral $(\mathrm{ML}) \pm 2.9$, dorsoventral (DV) dura -6.7 . Infusions were made using a $10 \mu \mathrm{l}, 26$ gauge Hamilton microsyringe (Fisher Scientific, Loughborough, UK) that was left in place for $4 \mathrm{~min}$ before and after the bolus infusion $(0.5 \mu \mathrm{l} /$ hemisphere $)$ of the toxin $(0.45 \mu \mathrm{g} / \mu \mathrm{l})$ or its vehicle, Dulbecco's saline (Book et al., 1994).

Intracortical probe implantation. Concentric design microdialysis probes made of Fitral 16 membrane and $2 \mathrm{~mm}$ in length were implanted at a $12^{\circ}$ angle into the area of the prefrontal/prelimbic cortex [for construction details, see Dalley et al. (1998)]. The following coordinates from Paxinos and Watson (1986) were used: AP bregma +3.0, ML \pm 1.2 $\mathrm{mm}$, DV dura $-4.0 \mathrm{~mm}$. Probes were affixed to the skull using skull screws and dental cement. Probes were perfused at a flow rate of 2.0 $\mu \mathrm{l} / \mathrm{min}$ with artificial CSF (aCSF) via a $2.5 \mathrm{ml}$ gas-tight syringe mounted on a microsyringe pump. The aCSF solution contained (in mM): 147 $\mathrm{NaCl}, 3.0 \mathrm{KCl}, 1.0 \mathrm{MgCl}_{2}, 1.3 \mathrm{CaCl}_{2}$, and 1.5 sodium phosphate buffer, $\mathrm{pH} 7.4$, with $50 \mathrm{~nm}$ neostigmine.

Neurochemical analyses. NA and ACh concentrations were determined in $10 \mu \mathrm{l}$ aliquots using HPLC and electrochemical detection. NA was separated at $22^{\circ} \mathrm{C}$ on a C18 analytical column $(100 \times 4.6 \mathrm{~mm}$ octadecyl silica $3 \mu \mathrm{m}$; Luna, Phenomenex, UK). The mobile phase, $\mathrm{pH}$ 5.0, consisted of sodium acetate $(2 \mathrm{gm} / 1)$, citric acid $(4 \mathrm{gm} / \mathrm{l}), 1$-octane sulfonic acid $(0.4 \mathrm{gm} / \mathrm{l})$, EDTA $(25 \mathrm{mg} / \mathrm{l})$, and methanol $(10 \%)$ and was delivered at $0.75 \mathrm{ml} / \mathrm{min}$. Detection limits of $1-2 \mathrm{fmol}$ were achieved using a coulometric electrode (5014B ESA) with working potentials set at -200 $\mathrm{mV}(\mathrm{E} 1)$ and $250 \mathrm{mV}(\mathrm{E} 2)$. ACh was resolved at $28^{\circ} \mathrm{C}$ on a microbore column $(530 \times 1 \mathrm{~mm}, 10 \mu \mathrm{m}$; BAS, Congleton, UK) and detected by the oxidation of $\mathrm{H}_{2} \mathrm{O}_{2}$ formed on a post-column IMER $(50 \times 1 \mathrm{~mm}$; Chrompack, Millharbour, UK) across a peroxidase-coated wired Uni-Jet electrode $(0 \mathrm{mV}$ relative to a platinum reference electrode; BAS). The mobile phase contained $9 \mathrm{gm} / 1 \mathrm{Na}_{2} \mathrm{HPO}_{4}, 0.58 \mathrm{gm} / 1 \mathrm{NaCl}$, and $5 \mathrm{ml} / 11 \%$ ProClin ISO reagent (BAS) reagent, $\mathrm{pH}$ 8.0. The flow rate was 120 $\mu \mathrm{l} / \mathrm{min}$, and the detection limit was $\sim 2 \mathrm{fmol} / 5 \mu \mathrm{l}$ injection. Data acquisition and peak integration were completed using Gynkosoft software (version 5.4).

Histological procedures. At the conclusion of the microdialysis experiment, rats were given a lethal dose of sodium pentobarbital (Euthatal, Vet Drug; $200 \mathrm{mg} \cdot \mathrm{ml}^{-1} \cdot \mathrm{kg}^{-1}$, i.p.) and transcardially perfused with cold 0.01 m PBS followed by $4 \%$ paraformaldehyde. Brains were cryoprotected overnight in a $20 \%$ sucrose solution and sectioned using a freezing microtome $(40 \mu \mathrm{m})$. Every fourth section was stained for choline acetyltransferase (ChAT)-positive cell bodies with the adjacent section stained for Nissl substance (cresyl violet). Briefly, sections were rinsed in Tris-buffered saline (TBS) and then placed in 3\% normal goat serum (NGS) in TBS for $1 \mathrm{hr}$. Without rinsing, sections were then incubated in primary antibody (dilution 1:2000) in TBS with $1 \%$ NGS and $0.2 \%$ Triton X-100 (rabbit anti-ChAT; Chemicon International, Harrow, UK) Sections remained in the primary antibody for $24 \mathrm{hr}$, after which they were rinsed three times for $10 \mathrm{~min}$ in TBS. They were then incubated with biotinylated anti-rabbit secondary antibody (dilution 1:200; Vector Labs, CA) in TBS with Triton X-100 and 1\% NGS. They were then rinsed three times for $10 \mathrm{~min}$ in TBS before being placed in Vectastain Elite $\mathrm{ABC}$ in TBS with Triton X-100 and 1\% NGS for $3 \mathrm{hr}$. This was 
8followed by three $10 \mathrm{~min}$ washes in TBS and two 5 min washes in Tris non-saline (TNS) before visualization in a solution of $10 \mathrm{mg}$ of diaminobenzidine (Sigma, Poole, UK) in $20 \mathrm{ml}$ TNS and $6.6 \mu \mathrm{l} \mathrm{H}_{2} \mathrm{O}_{2}$ for 2-8 min.

Neuronal specificity of $192 \mathrm{IgG}$-saporin. In a separate group of animals $(n=4)$ that had not been trained on the 5CSRTT, an assessment was made of the specificity of $192 \mathrm{IgG}$-saporin for cholinergic and parvalbumin (PARV)-containing GABAergic neurons of the basal forebrain. Animals were anesthetized with ketamine and xylazine and prepared for surgery as described previously. Unilateral infusions $(0.5 \mu \mathrm{l} / 2 \mathrm{~min})$ of 192 IgG-saporin, counterbalanced by hemisphere, were made into the $\mathrm{NBM}$ at concentrations of either $0.15 \mu \mathrm{g} / \mu \mathrm{l}(n=2)$ or $0.45 \mu \mathrm{g} / \mu \mathrm{l}$. An equal volume of Dulbecco's saline was infused into the contralateral hemisphere. The coordinates used were as described previously for SAP HIGH subjects. Ten days later, animals were killed by sodium pentobarbital overdose (Euthatal, Vet Drug; $200 \mathrm{mg} \cdot \mathrm{ml}^{-1} \cdot \mathrm{kg}^{-1}$, i.p.), and transcardially perfused with cold $0.01 \mathrm{M}$ PBS followed by $4 \%$ paraformaldehyde. Brains were cryoprotected overnight in a $20 \%$ sucrose solution and sectioned using a freezing microtome $(40 \mu \mathrm{m})$. Every fourth section was stained for PARV-positive cells with the adjacent section stained for ChAT, as described above. For PARV staining, sections were rinsed in TBS and then placed in 3\% NGS in TBS for $1 \mathrm{hr}$. Without rinsing, sections were then incubated in primary antibody (dilution 1:1000) in TBS with $0.2 \%$ Triton X-100 and $1 \%$ NGS (PARV antimouse; Chemicon International). Sections remained in the primary antibody for $24 \mathrm{hr}$ after which they were rinsed three times for 10 min with TBS. After this they were incubated with biotinylated anti-mouse secondary antibody (dilution 1:200; Vector Labs) in TBS with $0.2 \%$ Triton $\mathrm{X}-100$ and $1 \%$ NGS. They were then rinsed three times for $10 \mathrm{~min}$ in TBS and placed in Vectastain Elite ABC, in TBS with $1 \%$ NGS, for $2 \mathrm{hr}$. This was followed by three 10 min washes in TBS and two 5 min washes in TNS before visualization with diaminobenzidine.

Histological quantification. ChAT-immunoreactive (IR) and PARV-IR neurons were counted in the vertical diagonal band of Broca and nucleus basalis magnocellularis [ $\mathrm{Ch} 2$ and $\mathrm{Ch} 4$ regions, as designated by Mesulam et al. (1983a,b)] using a Leica DMRB (Leica, Milton-Keynes, UK) microscope. Every fourth section through the area of the vertical limb of the diagonal band (VDB) was collected, resulting in four to five sections per subject. The area of the decussation of the anterior commissure was used to define the anterior limit of the NBM region with the total number of ChAT-IR neurons counted in every fourth section (e.g., every $200 \mu \mathrm{m}$ ) for this and the subsequent seven sections. Cresyl violet staining was used to determine the extent of any general neuronal loss and gliosis in the region of the infusion.

\section{Experiment 2}

Twenty-four Lister hooded, male rats (Harlan, Olac, Bicester, UK) began the study. Two rats failed to reach behavioral criterion in the attentional task, and an additional six rats failed to complete the study because of an adverse reaction to anesthesia or damage to their cortical microdialysis probes that prohibited in vivo microdialysis.

Experimental design. Rats were randomly assigned to receive either vehicle or $0.15 \mu \mathrm{g} / \mu \mathrm{l}$ infusions $(0.5 \mu \mathrm{l} /$ hemisphere $)$ of $192 \mathrm{IgG}$-saporin (SAP LOW) into the NBM.

Post-surgical behavioral challenges. An array of behavioral challenges were used to vary the attentional demands of the task, including increased event rate (ITI $=2 \mathrm{sec} ; \mathrm{SD}=500 \mathrm{msec}$ ), increased event asynchrony (random ITI: $2,4,7,8 \mathrm{sec} ; \mathrm{SD}=500 \mathrm{msec}$ ), decreased event rate $(\mathrm{ITI}=10 \mathrm{sec} ; \mathrm{SD}=500 \mathrm{msec}$ ), or decreased stimulus duration (either 250 or $125 \mathrm{msec}$ : ITI $=5 \mathrm{sec}$ ). These testing sessions were separated by at least one standard testing session and compared against the standard testing session (ITI $=5.0 \mathrm{sec} ; \mathrm{SD}=500 \mathrm{msec}$ ) immediately before the challenge session. If performance on the day before and after the behavioral challenge was similar, another challenge test was scheduled. Otherwise, rats were given as many standard testing sessions as required to reestablish stable responding. After completion of the behavioral challenges, rats were implanted with intracortical microdialysis probes and tested on 2 consecutive days with an SD of 500 or $250 \mathrm{msec}$, ITI $=5.0 \mathrm{sec}$. This decrease in stimulus duration has been shown previously to impair accuracy in the 5CSRTT and increase the attentional demands of the task, but the rats maintain a relatively low level of omissions under these conditions. All other surgical, neurochemical, and histological procedures were identical to those used in experiment 1.

Statistical analyses. Data analyses were performed using SPSS version 10.0 (SPSS, Chicago, IL). Histological analyses revealed that one SAP LOW animal had a unilateral lesion, resulting in a sample of seven
Table 1. The effects of intrabasalis saporin infusions on the number of ChAT-IR neurons in the NBM and VDB (mean \pm SEM)

\begin{tabular}{lllll} 
& $\begin{array}{l}\text { SHAM } \\
(\text { experiment 1) }\end{array}$ & \multicolumn{1}{l}{ HIGH } & $\begin{array}{l}\text { SHAM } \\
\text { (experiment 2) }\end{array}$ & $\begin{array}{l}\text { SAP } \\
\text { LOW }\end{array}$ \\
\hline NBM & $1219 \pm 133$ & $276 \pm 30^{*}$ & $945 \pm 81$ & $538 \pm 80^{*}$ \\
VDB & $232 \pm 37$ & $57 \pm 13^{*}$ & $209 \pm 20$ & $179 \pm 37$
\end{tabular}

Chat-IR cells in NBM and VDB of SHAM and SAP rats. Asterisks indicate values that are significantly lower than controls $\left({ }^{*} p<0.05\right)$. Note that although the lesion of SAP LOW rats was limited to the region of the NBM, the higher dose used in the SAP HIGH group produced additional damage to VDB ChAT-IR neurons.

lesioned animals. Therefore a random sample of seven of eight of the SHAM rats in each study was made using SPSS to produce equal numbers in each group as recommended by Keppel (1991). Accuracy data were angularly transformed as recommended by Zar (1974). Main analyses consisted of independent mixed-factor ANOVAs on the effects of the between-subject factor LESION (two levels), by the mean performance of post-operative days $1-5$ versus days $6-10$ (2) by blocks of 25 trials (four blocks per testing session). The factor blocks of trials (BLK) assesses the effects of time on task because prolonged time on task increases the sustained attentional demands in both humans and rats (Parasuraman et al., 1987; Parasuraman and Giambra, 1991; McGaughy and Sarter, 1995). For behavioral challenge days, the performance on the day before the challenge session was used as a baseline. These analyses then became LESION (2) $\times$ CHALLENGE (2) $\times$ BLK (4). Behavioral data collected on microdialysis days were analyzed by three-way ANOVA of LESION $\times$ DAY (collection day 1 vs 2$) \times$ BLOCK (6). The six test blocks correlated with the collection of each dialysate sample. The absolute levels in femtomole per sample of ACh and NA were analyzed in independent ANOVAs. Analysis of basal efflux (mean of the three samples immediately before task onset) was made using a two-way ANOVA of LESION $\times$ DAY. This basal sample was then compared with the subsequent six samples collected after task onset in a mixed-factors ANOVA of LESION $\times$ DAY $\times$ BLOCK (7). Multiple comparisons were conducted using modified Bonferroni corrections to control the familywise $\alpha$ level. Additionally, Huynh-Feldt correction $\epsilon$ values are given and used to correct the degrees of freedom in the case of a violation of sphericity as recommended by Keppel (1991). In accordance with Greenwald et al. (1996), the exact $p$ values are reported.

The relationship of the extent of the damage as indicated by ChAT-IR neurons in the VDB and NBM regions for subjects from both studies was correlated with measures of task performance, including accuracy, omissions, and premature and perseverative responses using Pearson's correlation coefficient, $r$. Because the number of ChAT-IR neurons in the VDB and NBM were necessarily related, partial correlation coefficients were calculated so that the correlation of ChAT-IR in the VDB was controlled for the effect of ChAT-IR in the NBM, and vice versa. Additionally, cortical ACh efflux from day 1 during the task was correlated with measures of behavioral performance and ChAT-IR in the basal forebrain using Pearson's correlation coefficients. Correlations of cortical ACh efflux with performance were similarly controlled for changes in NA efflux using partial correlation.

\section{RESULTS}

\section{Experiment 1}

Histological analysis of SAP HIGH lesions of the NBM region ChAT-IR neurons were found throughout the VDB and NBM. Independent $t$ tests revealed that ChAT-IR neurons in both the $\mathrm{NBM}$ region $\left(t_{(12)}=4.48 ; p=0.001\right)$ and the $\operatorname{VDB}\left(t_{(12)}=6.93\right.$; $p<0.001$ ) were significantly lower in SAP HIGH rats (Table 1 ). There was no evidence of gross, nonselective neuronal destruction, for example, cavitation (Fig. 1). Additional examination of the effect of the same high dose of $192 \mathrm{IgG}$-saporin on untrained rats revealed that parvalbumin-containing cells within the basal forebrain were generally unaffected by the $192 \mathrm{IgG}$-saporin infusion (Fig. 2). 

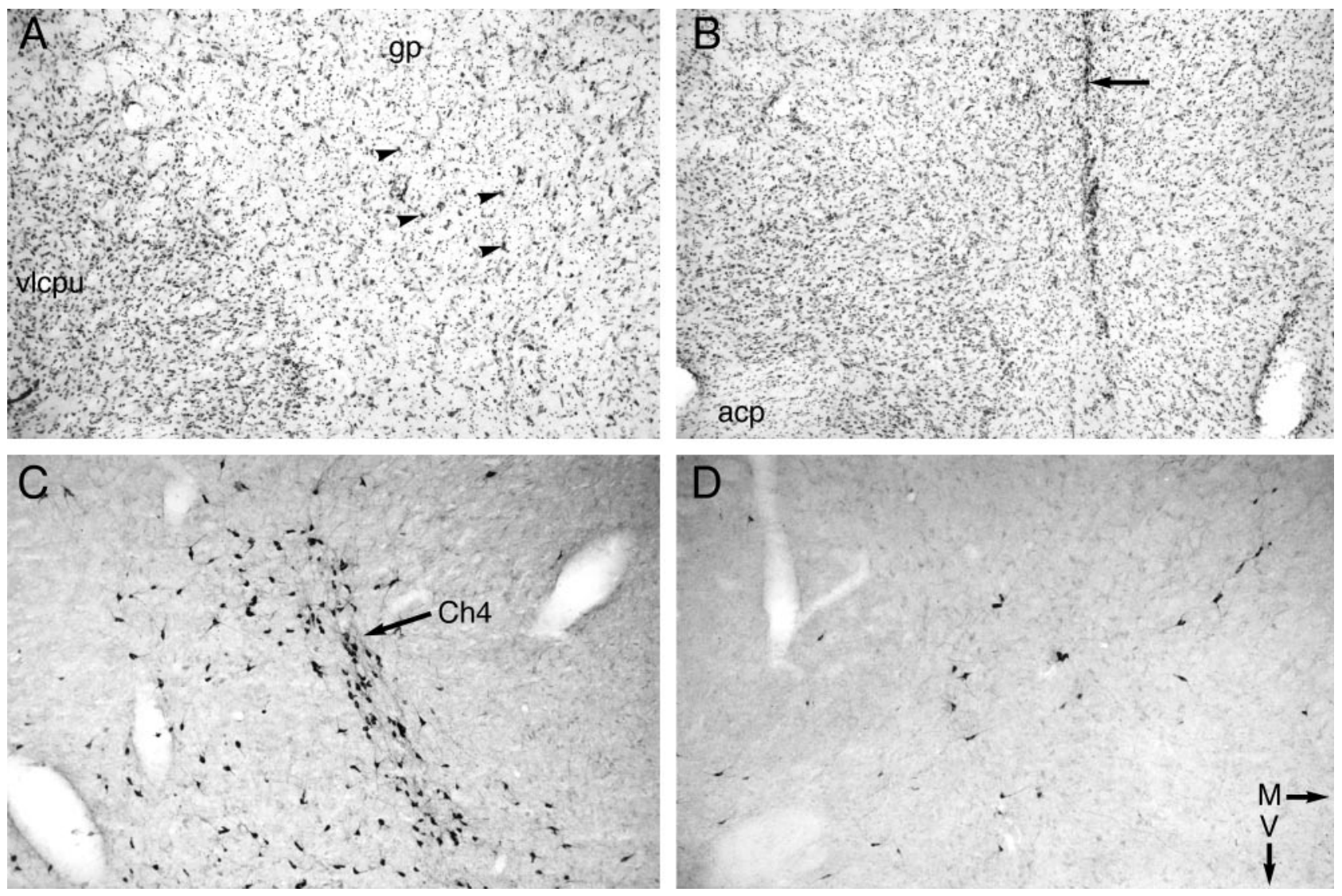

Figure 1. A, B, Photomicrographs of sections of the basal forebrain stained with cresyl violet showing the effects of inf usion of 192 IgG-saporin (SAP $\mathrm{HIGH})(B)$ compared with a control subject that received infusions of vehicle at the same coordinates $(A)$. The magnocellular, hyperchromatic neurons of the nucleus basalis neurons that can be seen in $A$ (arrowheads) are markedly reduced in number in the SAP-infused brain (B), despite otherwise excellent preservation of neurons in the globus pallidus $(g p)$ and ventrolateral caudate-putamen $(v l c p u)$. The arrow in $B$ indicates the glial-rich site of the cannula tract through which $192 \mathrm{IgG}$-saporin inf usions were made. $C, D$, Sections adjacent to $A$ and $B$, respectively, stained using antibodies to choline acetyltransferase to reveal the magnocellular neurons of the $C h 4$ cell group $(C$, arrow). The marked reduction in the number of cholinergic neurons can be seen in the 192 IgG-saporin-infused brain (SAP HIGH) (D). acp, Posterior limb of the anterior commissure; $M$, medial; $V$, ventral.

\section{Behavioral effects of SAP HIGH lesions}

Over the $10 \mathrm{~d}$ post-operative period, lesions of the nucleus basalis region produced profound behavioral impairments in overall performance of the 5CSRTT, with decreases in accuracy $\left(F_{(1,12)}=45.71 ; p<0.001\right)$ and increases in percentage of trials omitted $\left(F_{(1,12)}=8.62 ; p=0.012\right)$, correct response latencies $\left(F_{(1,12)}=18.39 ; p=0.001\right)$, number of perseverative $\left(F_{(1,12)}=5.88 ; p=0.03\right)$, and premature responses $\left(F_{(1,12)}=\right.$ $6.47 ; p=0.03)$. Specifically, accuracy of responding and the latency for correct responses in only the SAP HIGH rats decreased significantly by test days $6-10$ relative to days $1-5$ (accuracy: $t_{(6)}=3.67, p=0.01$; correct latencies: $t_{(6)}=-4.10$, $p=0.006)$. SAP HIGH rats made more perseverative responses than the control group, but only in later stages of retraining $\left(t_{(12)}=-2.92 ; p=0.013\right.$; corrected $\left.\alpha=0.025\right)$. Figure 3 shows how these behavioral effects evolved as a function of the post-operative period.

\section{Effects of increased stimulus duration}

Increasing the target stimulus duration ameliorated the accuracy deficits of SAP HIGH rats $\left(\mathrm{LESION} \times \mathrm{SD}: F_{(1,12)}=11.35 ; \epsilon=\right.$ $1.0 ; p=0.006)$. Student's $t$ tests revealed that although SAP HIGH rats were significantly impaired under baseline conditions $\left(t_{(12)}=6.28 ; p<0.001\right)$ (Fig. 3, black bars), there was no difference in the accuracy between the groups when tested with a
$5000 \mathrm{msec} \operatorname{SD}\left(t_{(12)}=1.6 ; p=0.14\right)($ Fig.4, white bars $)$. Together with the lack of effects on magazine latency throughout the post-operative testing period, this result indicates that the SAP HIGH rats did not exhibit motivational deficits.

\section{Intracortical microdialysis (SAP HIGH)}

The marked attentional impairments observed in SAP HIGH animals were also evident during subsequent in vivo microdialysis experiments (data not shown), with accuracy being decreased $\left(F_{(1,12)}=24.94 ; p<0.001\right)$ and premature responses increased $\left(F_{(1,12)}=7.51 ; p=0.02\right)$. Percentage accuracy and premature responses in sham and SAP HIGH rats were $86 \pm 2$ and $65 \pm 4$ and $3.1 \pm 0.6$ and $7.5 \pm 1.4$, respectively. SAP HIGH rats showed significantly reduced levels of cortical ACh both before $\left(F_{(1,12)}=\right.$ $9.99 ; p=0.01)$ and during $\left(F_{(1,12)}=15.16 ; p=0.002\right)$ attentional testing. Overall, task onset coincided with an increase in cortical ACh efflux $\left(F_{(6,72)}=8.26 ; p=0.001 ; \epsilon=0.41\right)$. Basal levels of $\mathrm{ACh}$ (femtomoles/10 min) in SHAM and SAP HIGH animals were $289 \pm 49$ and $125 \pm 17$, respectively. Thirty minutes after the initiation of the task, levels increased to $416 \pm 45$ and $178 \pm$ 19 , respectively. Neither basal efflux $\left(F_{(1,12)}=1.37 ; p=0.3\right)$ nor stimulated efflux $\left(F_{(1,12)}=0.04 ; p=0.84\right)$ of NA differed between SHAM (4.21 $\pm 0.96 \mathrm{fmol} / 10 \mathrm{~min})$ and SAP HIGH $(4.93 \pm 1.12$ fmol/10 min) rats. These data show that performance of the 5CSRTT is associated with sustained elevations in cortical ACh 

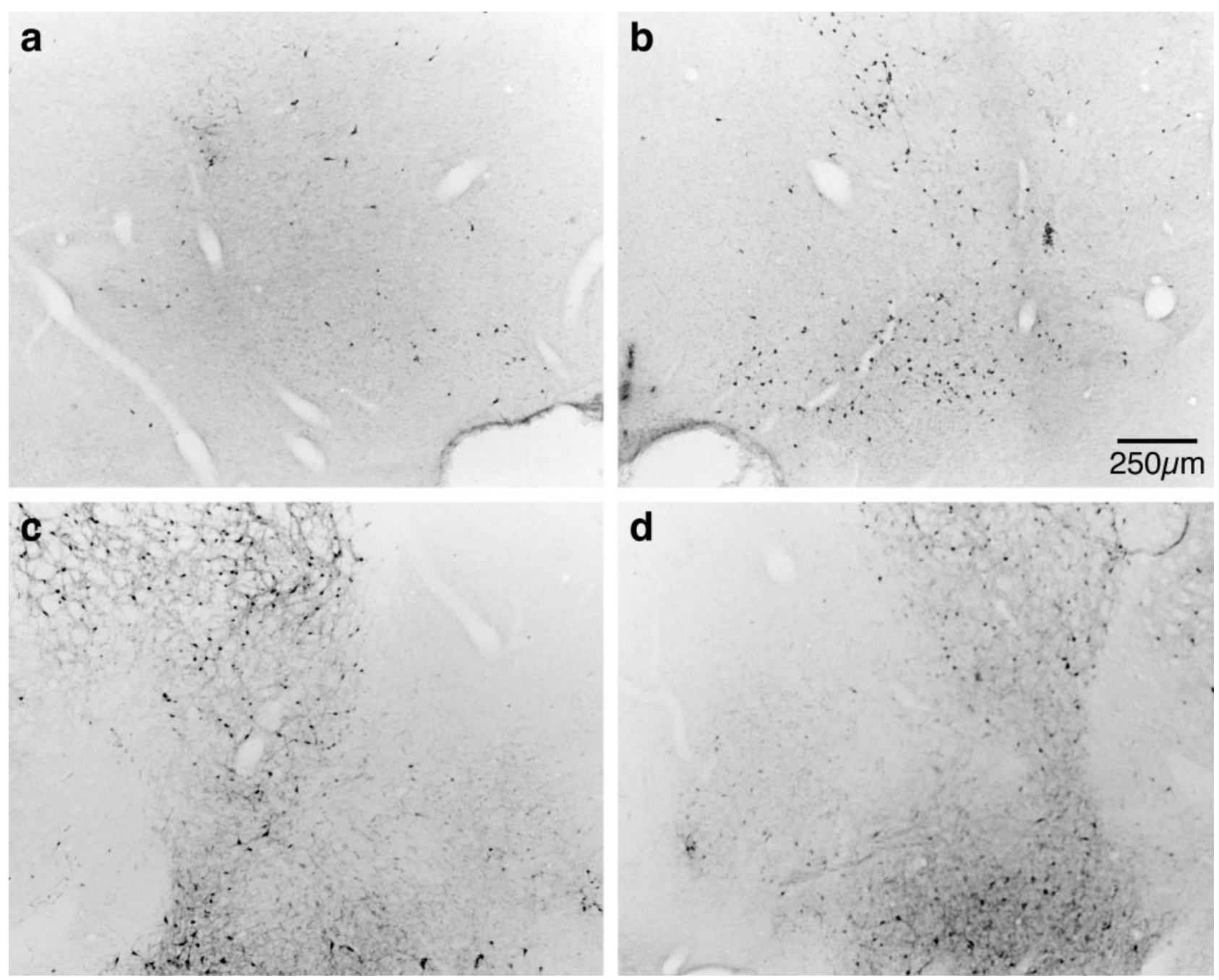

Figure 2. Representative photomicrographs showing ChAT-IR $(a, b)$ and PARV-IR $(c, d)$ neurons in the basal forebrain of SHAM (right-hand panels) and SAP HIGH (left-hand panels) lesioned rats. It can be seen that the magnocellular ChAT-IR neurons of the Ch4 cell group (nucleus basalis magnocellularis) are greatly reduced in number after intrabasalis infusions of $192 \mathrm{IgG}$-saporin. By contrast, parvalbumin-containing neurons are unaffected by immunotoxin infusions in this region where cholinergic neurons are lost (compare $a, c)$.

efflux, in agreement with other reports (Passetti et al., 2000; Dalley et al., 2001), and that 192 IgG-saporin-induced lesions of the NBM produce a relatively selective depletion of ACh in the medial prefrontal cortex.

\section{Experiment 2}

Histological analyses of SAP LOW NBM lesions

Independent $t$ tests revealed that ChAT-IR neurons were significantly reduced in the NBM region $\left(t_{(12)}=3.57 ; p=0.004\right)$ but not the VDB region $\left(t_{(12)}=0.72 ; p=1.49\right)$ of SAP LOW lesioned rats (Table 1). Qualitative analysis revealed minimal gliosis and an apparent preseveration of noncholinergic neurons in the NBM, as reported previously (Baxter et al., 1995; Chiba et al., 1995; Wrenn et al., 1999).

\section{Baseline assessment}

SAP LOW rats were significantly impaired in choice accuracy relative to SHAM animals throughout the $10 \mathrm{~d}$ post-operative period $\left(F_{(1,12)}=19.77 ; p=0.001\right)($ Table 2$)$. This deficit was accompanied by an increase in premature responses $\left(F_{(1,12)}=\right.$ $6.78 ; p=0.02)$, but only during block 1 of test days $1-5$ $\left(\right.$ LESION $\times$ BLOCK $\times$ RECOVERY: $F_{(3,36)}=4.46, p=$ $0.009, \epsilon=0.97$; days $1-5: t_{(12)}=-3.44 ; p=0.005$; corrected $\alpha=0.019$ ). No other behavioral differences were observed (all $p>0.06)$.

\section{Testing under differential attentional demands}

Increased event rate ( 2 sec intertrial interval). Increasing the event rate of the visual targets dissociated further the performance of SAP LOW and SHAM groups as a function of test block $\left(F_{(3,36)}\right.$ $=3.51 ; p=0.03 ; \epsilon=0.87)$. Under standard testing conditions, the percentage accuracy of SAP LOW animals was significantly lower than SHAM animals in blocks 3 and 4 (both $p<0.002$; corrected $\alpha=0.019$; means \pm SEM blocks 3 and 4 , respectively, SHAM: $97 \pm 1,98 \pm 1$; SAP LOW: $88 \pm 3,84 \pm 2$ ). When the attentional demands of increased event rate were added, the performance of the SAP LOW animals fell below that of the SHAM animals as early as block 2 of testing $\left(t_{(12)}=2.96 ; p=0.012\right.$; SHAM: $93 \pm$ 0.06; SAP LOW: $74 \pm 0.06$ ).

Increased event asynchrony (variable intertrial interval). Changes in event asynchrony did not change accuracy, perseverative responses, or response latency (all $p>0.08$ ). Omissions were 

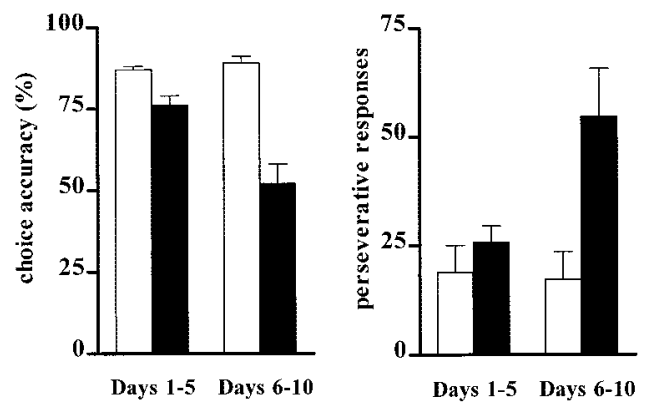

Figure 3. Effects of intrabasalis SAP $(0.45 \mu \mathrm{g} / \mu \mathrm{l})$ on accuracy (left panel) and perseverative responses (right panel) on days 1-5 (left bars) and days 6-10 (right bars) of post-operative performance. SHAM animals (white bars) show a similar level of performance across all stages of retraining, whereas SAP HIGH rats (black bars) become progressively more impaired by days 6-10. Perseverative responses did not differ between the two groups on days $1-5$; however, by day 6-10 these responses increased markedly in SAP HIGH animals.

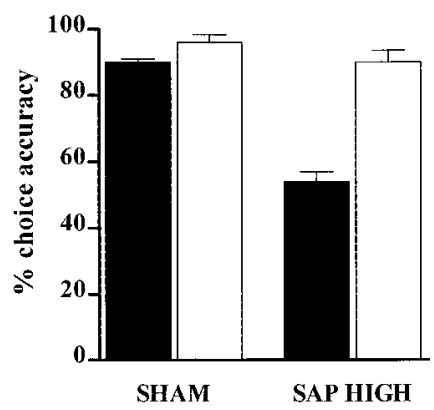

Figure 4. Accuracy of SHAM and SAP HIGH rats on the 5CSRTT during a standard test session $(\mathrm{SD}=500 \mathrm{msec}$; black bars $)$ and during a session with a longer SD (5000 msec; white bars). It can be seen that the profound deficit in stimulus discriminability is reversed by the introduction of the longer stimulus duration.

elevated in both groups $\left(F_{(1,12)}=25.31 ; p<0.001 ; \epsilon=1.0\right)$, although SAP LOW rats showed a significantly greater increase $\left(F_{(1,12)}=7.44 ; p=0.018 ; \epsilon=1.0\right)$. Premature responses were also increased $\left(F_{(1,12)}=11.38 ; p=0.006 ; \epsilon=1.0\right)$ but not differentially between the groups $\left(F_{(1,12)}=0.87 ; p=0.37\right)$. No other changes in performance were found (all $p>0.06$ ).

Decreased event rate (10 sec intertrial interval). Reductions in event rate failed to differentiate the performance of SAP LOW and SHAM animals. The lower event rate did not change accu$\operatorname{racy}\left(F_{(1,12)}=4.06 ; p=0.07, \epsilon=1.0\right)$ but did increase the percentage of trials omitted $\left(F_{(1,12)}=5.64 ; p=0.04 ; \epsilon=1.0\right)$ and the number of premature responses $\left(F_{(1,12)}=30.93 ; p<0.001\right.$; $\epsilon=1.0)$ for all rats. This manipulation produced no other effects on the rats' performance.
Decreased stimulus duration. SAP LOW and SHAM rats performed in a similar manner when tested with decreased SD. Decreasing the SD to either 250 or $125 \mathrm{msec}$ decreased the accuracy of all rats (SD 250: $F_{(1,12)}=14.44, p<0.003$; SD 125: $F_{(1,12)}=249.53, p<0.001 ;$ all $\left.\epsilon=1.0\right)$ and increased the percentage of trials omitted (SD 250: $F_{(1,12)}=6.20, p<0.02$; SD 125: $F_{(1,12)}=13.64, p=0.003$; both $\left.\epsilon=1.0\right)$ relative to their performance with the $500 \mathrm{msec}$ SD. Testing with the $250 \mathrm{msec}$ stimulus duration also decreased correct response latencies $\left(F_{(1,14)}=7.37 ; p<0.02 ; \epsilon=1.0\right)$ and decreased perseverative responses $\left(F_{(1,12)}=4.96 ; p=0.05 ; \epsilon=1.0\right)$ in all rats. Testing with the $125 \mathrm{msec}$ stimulus duration increased premature responses $\left(F_{(1,12)}=6.26 ; p=0.03 ; \epsilon=1.0\right)$ in all rats.

\section{Intracortical microdialysis (SAP LOW)}

The attentional performance of SAP LOW rats did not differ from that of SHAM animals for all measures (all $F<1.0$; NS), except for perseverative errors, which on day 1 were higher in lesioned animals $\left(\mathrm{LESION} \times \mathrm{DAY}: F_{(1,12)}=6.49, p=0.03, \epsilon=\right.$ 1.0 ; DAY $\left.1: t_{(6)}=3.55 ; p=0.012\right)$ than DAY $2\left(t_{(6)}=-1.06 ; p=\right.$ $0.33)$. The number of trials completed during the 60 min session was high (mean \pm SEM: $262 \pm 6)$ and did not differ between the two groups $\left(F_{(1,12)}=1.77 ; p=0.21\right)$. Choice accuracy and premature responding $( \pm$ SEM) in SHAM and lesioned rats was $75 \pm 2$ and $72 \pm 3$ and $2.2 \pm 0.3$ and $2.3 \pm 0.5$, respectively.

Figure 5 shows the temporal profile of ACh efflux in the medial prefrontal cortex of SHAM and SAP LOW subjects during attentional performance over 2 consecutive days. Basal ACh efflux (femtomoles/10 min) was significantly higher in SAP LOW subjects $(249 \pm 27)$ than SHAM animals $(154 \pm 35)$ on the first day of testing $\left(F_{(1,12)}=4.85 ; p=0.05\right)$. Cortical ACh efflux increased significantly in both groups after task onset $\left(F_{(6,72)}=31.37 ; p<\right.$ $0.001 ; \epsilon=0.44)$, the magnitude being similar in both groups $\left(F_{(6,72)}=2.54 ; p=0.08 ; \epsilon=0.44\right)$ on each day $\left(F_{(1,12)}=0.90 ; p=\right.$ $0.36 ; \epsilon=1.0)$.

Basal NA efflux (femtomoles/10 min \pm SEM) did not differ between SHAM $(4.7 \pm 0.8)$ and SAP LOW $(4.6 \pm 0.6)$ subjects on either day (LESION: $F_{(1,12)}=0.05, p=0.82$; LESION $\times$ DAY: $\left.F_{(1,12)}=0.01, p=0.92 ; \epsilon=1.0\right)$. Stimulated NA efflux coincident with task onset did not differ between the groups of animals $\left(F_{(1,12)}=0.072 ; p=0.79\right)$.

\section{Correlations between ChAT immunoreactivity, cortical ACh} efflux, and task performance

The results of the correlational analyses between ChAT-IR in the NBM, cortical ACh efflux, and attentional performance are shown in Figure 6 and Table 3. The number of ChAT-IR neurons in the NBM was significantly and positively correlated with accuracy $(r=0.60 ; p=0.002)$ but was not correlated with omissions or premature or perseverative responses (all $p>0.09$ ). By contrast,

Table 2. Effects of intrabasalis saporin infusions on performance of the five-choice serial reaction time task (mean \pm SEM)

\begin{tabular}{lcccc} 
& SHAM (experiment 1) & SAP HIGH & SHAM (experiment 2) & SAP LOW \\
\hline Accuracy & $0.88 \pm 0.01$ & $0.64 \pm 0.03^{*}$ & $0.89 \pm 0.03$ & $0.83 \pm 0.03^{*}$ \\
Omissions & $0.16 \pm 0.04$ & $0.35 \pm 0.05^{*}$ & $18.84 \pm 3.56$ & $0.23 \pm 0.06$ \\
Perseveration & $17.14 \pm 5.45$ & $40.14 \pm 6.70^{*}$ & $5.00 \pm 1.00$ & $27.68 \pm 10.48$ \\
Prematures & $14.59 \pm 3.23$ & $46.33 \pm 12.05^{*}$ & $0.59 \pm 0.04$ & $13.29 \pm 2.92^{*}$ \\
Correct latencies & $0.56 \pm 0.02$ & $0.78 \pm 0.06^{*}$ & $0.56 \pm 0.03$
\end{tabular}

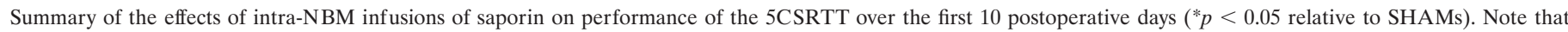

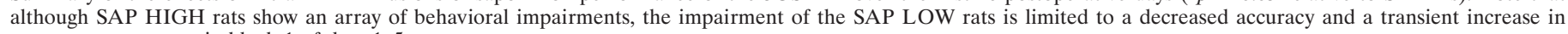
premature responses in block 1 of days $1-5$. 

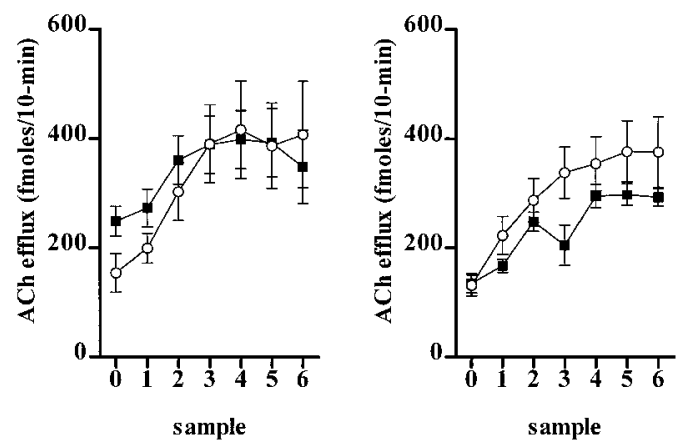

Figure 5. Cortical ACh efflux in SHAM $(\bigcirc)$ and SAP LOW $(\square)$ subjects during sustained attentional performance over 2 consecutive days (day 1 , left panel; day 2, right panel). The data are absolute levels of ACh expressed as femtomoles/10 min $( \pm \mathrm{SEM})$. Sample 0 represents the final basal sample before task onset. The remaining levels are successive 10 min samples collected concurrently during behavioral performance.

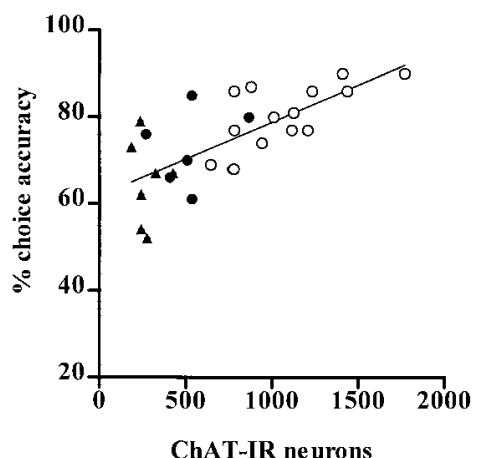

Figure 6. Correlation plots showing the relationship between the number of ChAT-immunoreactive neurons in the NBM with choice accuracy on the 5CSRTT. Linear regression of the data for SHAM $(\bigcirc)$, SAP LOW $(\bullet)$, and SAP HIGH (४) animals revealed a strong positive association between NBM ChAT-IR neurons and accuracy.

\begin{tabular}{|c|c|c|c|}
\hline & NBM & VDB & ACh efflux \\
\hline Accuracy & $r=0.60^{*}$ & $r=0.36$ & $r=0.54^{*}$ \\
\hline Omissions & $r=-0.20$ & $r=0.04$ & $r=-0.36$ \\
\hline Premature responses & $r=-0.17$ & $r=-0.28$ & $r=-0.20$ \\
\hline
\end{tabular}

Correlations (Pearson's $r$ values) between behavioral performance, cortical ACh efflux during task engagement, and the number of ChAT-IR neurons in the NBM and VDB $\left({ }^{*} p<0.05\right)$. Note that accuracy correlates significantly with ChAT-IR cells in the NBM as well as cortical ACh efflux measured during task performance.

the number of ChAT-IR neurons in the VDB did not correlate significantly with any aspect of task performance $(p>0.05)$.

Task-related changes in ACh release correlated positively with accuracy $(r=0.54 ; p=0.003)$ but not with premature responses or omissions (all $p>0.05$ ). Cortical ACh during the attentional task correlated positively and significantly with the number of ChAT-IR neurons in the NBM $(r=0.41 ; p=0.03)$ but not in the $\operatorname{VDB}(p>0.05)$.

\section{DISCUSSION}

Intrabasalis infusions of 192 IgG-saporin produced dosedependent effects on attentional accuracy that correlated significantly, both with cholinergic cell loss in the NBM and with reductions in ACh efflux in the prefrontal cortex. This is among the clearest evidence to date of a systematic relationship between indices of cholinergic function and attentional performance. The higher dose of toxin produced more general behavioral impairments, whereas the lowest dose produced a specific impairment in accuracy that sometimes varied with time on task, and a transient increase in premature responses that dissipated by day 6 of behavioral retraining. The smaller dose of saporin significantly increased basal levels of ACh efflux in the prefrontal cortex, with no significant decrease in task-related ACh efflux, suggesting that the basal forebrain cholinergic system may compensate for limited amounts of damage.

\section{Behavioral deficits resulting from low- and high-dose saporin}

The SAP HIGH group differed from SAP LOW rats primarily in the extent of damage to the basal forebrain. Although high doses of the toxin produce concomitant lesions of the VDB [see also Stoehr et al. (1997)], such damage did not correlate significantly with any aspect of behavioral performance. The lack of significant correlation, although not conclusive, agrees with previous data showing no causal relationship between damage targeted to the VDB and septum on performance of the same attentional task (Muir et al., 1996b; Lehmann et al., 2001).

The accuracy of the SAP HIGH rats declined progressively over test days in parallel with increases in perseverative responses in the latter stages of post-surgical retraining. This combination of increased perseverative responding with already high levels of premature responding is consistent with a disruption in inhibitory response control resulting from aberrant modulation of the prefrontal cortex (Miner et al., 1997) and further exacerbates the response accuracy deficit. Lengthening the target stimulus duration alleviated these behavioral deficits, suggesting that they arise from attentional rather than motivational impairments (Muir et al., 1994). The precise mechanisms underlying the relatively late onset of the nonspecific behavioral effects are not known. It is possible that they reflect relatively distinct neuronal damage to noncholinergic neurons. Inspection of Figure 6 suggests that a subset of SAP HIGH animals had a disproportionately impaired response accuracy score, which could be consistent with some additional nonspecific cell loss. However, there were no gross signs of nonselective damage such as cavitation in this group, and although parvalbumin staining did not reveal evidence of GABAergic neuronal loss in the basal forebrain, we cannot exclude the possibility of nonselectivity at this high dose of saporin.

Further evidence for the behavioral specificity of these lesion effects is provided by studies that have found either no effect on mnemonic function after intra-NBM infusions of $192 \mathrm{IgG}$ saporin (Torres et al., 1994) or have reported delay-independent effects hypothesized to be attentional in nature (Baxter et al., 1995). Recent reports have suggested that cavitation within the basal forebrain as a result of an extended post-lesioning period necessarily precedes deficits in mnemonic function (Perry et al., 2001). However, it remains unclear whether cavitation was simply related to the time after lesion or was the result of the high doses and volumes of the toxin used by Perry et al. (2001).

In contrast to the SAP HIGH group, SAP LOW rats were only sensitive to changes in task parameters that have been shown to increase sustained attentional demands, namely time on task and high event rate. These effects are consistent with previous data in rats (McGaughy and Sarter, 1995) and in humans (Parasuraman et al., 1987; Parasuraman and Giambra, 1991). Although previous 
work reported that NBM lesioned rats are sensitive to shortened stimulus durations (Muir et al., 1994), the present study failed to show such an effect in SAP LOW rats, probably because the damage was less extensive than in the study by Muir et al. (1994). Manipulations of other task parameters, such as increasing event asynchrony, also failed to differentiate the accuracy of lesioned and SHAM rats, similar to what has been reported with less selective lesions of the cholinergic system (Muir et al., 1995). The effects of restricted lesions in the SAP LOW group resembled those of excitotoxic lesions restricted to the area of the medial frontal cortex (Muir et al., 1996a), with the exception that no increase in perseverative responding was found in the SAP LOW group.

Previous work by Waite et al. (1999) investigated the effects of intracerebroventricular infusions of SAP on performance in the 5CSRTT. To control for the effects of cerebellar damage produced by this lesioning method, an additional group that sustained only cerebellar Purkinje cell damage (OX7-saporin) was included. An increase in the size of the visual search array did selectively impair performance of the $192 \mathrm{IgG}$-saporin groups, and damage to the cortical cholinergic system alone was sufficient to produce this deficit. Although manipulation of other spatiotemporal aspects of the task produced deficits in the Waite et al., (1999) study, it could not be determined whether the resulting deficits were caused by neuronal damage within the cholinergic basal forebrain or by the additional cerebellar Purkinje fiber loss.

\section{Neurochemical analyses}

The accuracy of rats during behavioral microdialysis correlated positively with the absolute level of cortical ACh but not cortical NA. This correlation provides additional, novel evidence for a critical role for cortical $\mathrm{ACh}$ in the successful maintenance of sustained attention. However, a simple relationship between accuracy and cortical ACh should not be assumed. Previous work has shown that changes in the firing of medial frontal cortex neurons are correlated with the demands placed on sustained attentional resources. Firing rates increase during conditions of heightened attentional demand (e.g., after presentation of a distractor stimulus) despite decreases in accuracy (Gill et al., 2000). The present results agree with those showing that attentional testing is correlated with elevated cortical ACh efflux, even in the presence of decreased accuracy (Himmelheber et al., 2000).

Small SAP-induced lesions of the frontal cortex produce decreases in basal but not stimulated cortical cholinergic efflux (Fadel et al., 1996). In the current study, SAP LOW NBM lesioned rats showed transient increases in basal, but not taskrelated, frontal ACh efflux. Several factors may account for these differences. First, the intracortical infusion of the immunotoxin in Fadel et al. (1996) likely produced the greatest damage in the vicinity of the cortical probe, whereas in the current study cortical cholinergic dennervation was more diffuse and not necessarily greatest in the area sampled by the microdialysis probe. Second, basal ACh efflux in the present study may have been higher on DAY 1 than in Fadel et al. (1996), because the rats were not habituated to the conditions of in vivo microdialysis. This possible reactivity to novelty, however, cannot explain the attentional impairments shown by SAP LOW rats in the 5CSRTT, because they showed no impairments under other novel testing conditions (i.e., increased event asynchrony and shortened SD).

No differences were found in basal or stimulated NA efflux in SAP lesioned rats. Although SAP HIGH lesioned rats showed similar levels of NA efflux over the course of the testing session, their behavioral impairments persisted, indicating that the coeruleocortical NA system does not compensate for loss of cholinergic function in the cortex. Moreover, cortical NA was found not to correlate with any aspect of performance in the 5CSRTT (data not shown). Although NA has been implicated in attentional function (Aston-Jones et al., 1994; Bunsey and Strupp, 1995; Coull, 1998), performance of the 5CSRTT (Carli et al., 1983) and other sustained attentional tasks (McGaughy et al., 1997) is less dependent on the integrity of the coeruleocortical NA system.

In summary, the present data demonstrate that the cognitive effects of a compromised basal forebrain-cortical cholinergic system are dependent on the attentional demands placed on the subject, the number of neurons surviving in the NBM, and the responsivity of ACh function in the medial frontal cortex. Attentional impairments during in vivo microdialysis were significantly correlated with decreased ACh efflux in rats with more extensive lesions of the NBM. Incomplete lesions of the NBM produced no change in cortical $\mathrm{ACh}$ efflux during attentional testing and no impairment in accuracy, suggesting that the basal forebraincortical system effectively compensates for the loss of ChAT-IR neurons in the NBM to maintain attentional function. However, there are probably limits to the extent of this behavioral compensation. SAP LOW rats were still unable to maintain accuracy when a high event rate increased attentional demands. This effect was present regardless of the time after lesioning, suggesting that it was not simply present before the occurrence of compensatory mechanisms. Had the microdialysis experiment been performed during high event rates, it might have been hypothesized that cortical ACh efflux of SAP LOW rats would have been significantly lower than SHAM rats. The high omission rate produced by increased event rate precluded this test, but future studies could investigate the effects of manipulating event rate within one block of the testing session. These data provide new evidence to support the crucial relationship of the integrity of the NBM to attentional function and cortical ACh efflux. Furthermore, these data emphasize that the critical assessment of the neurochemical and cognitive effects of 192 IgG-saporin has to take into account not only the extent and specificity of damage to the NBM, but also the cognitive demands placed on the subject.

\section{REFERENCES}

Aston-Jones G, Rajkowski J, Kubiak P, Alexinsky T (1994) Locus coeruleus neurons in monkey are selectively activated by attended cues in a vigilance task. J Neurosci 14:4467-4480.

Aston-Jones G, Rajkowski J, Cohen J (2000) Locus coeruleus and regulation of behavioral flexibility and attention. Prog Brain Res 126:165-182.

Baunez C, Robbins TW (1997) Bilateral lesions of the subthalamic nucleus induce multiple deficits in an attentional task in rats. Eur J Neurosci 9:2086-2099.

Baxter M, Bucci DJ, Gorman LK, Wiley RG, Gallagher M (1995) Selective immunotoxic lesions of basal forebrain cholinergic cells: effects on learning and memory in rats. Behav Neurosci 109:714-722.

Book AA, Wiley RG, Schweitzer JB (1994) 192 IgG-saporin: I. Specific lethality for cholinergic neurons in the basal forebrain of the rat. $J$ Neuropathol Exp Neurol 53:95-102.

Bunsey M, Strupp BJ (1995) Specific effects of idazoxan in a distraction task: evidence that endogenous norepinephrine plays a role in selective attention in rats. Behav Neurosci 109:903-911.

Carli M, Robbins TW, Evenden JL, Everitt BJ (1983) Effects of lesions to ascending noradrenergic neurons on performance of a 5-choice serial reaction time task in rats: implications for theories of dorsal noradrenergic bundle function based on selective attention and arousal. Behav Brain Res 9:361-380.

Chiba A, Bucci DJ, Holland PC, Gallagher M (1995) Basal forebrain cholinergic lesions disrupt increments but not decrements in conditioned stimulus processing. J Neurosci 15:7315-7322.

Coull J (1998) Neural correlates of attention and arousal: insights from electrophysiology, functional neuroimaging and psychopharmacology.
Prog Neurobiol 55:343-361. 
Dalley JW, Parker CA, Wulfert E, Hudson AL, Nutt DJ (1998) Potentiation of barbiturate-induced alterations in presynaptic noradrenergic function in rat frontal cortex by imidazol(in)e alpha2-adrenoceptor agonists. Br J Pharmacol 125:441-446.

Dalley JW, McGaughy J, O'Connell MT, Cardinal RN, Levita L, Robbins TW (2001) Distinct changes in cortical acetylcholine and noradrenaline efflux during contingent and non-contingent performance of a visual attentional task. J Neurosci 21:4908-4914.

Detari L (2000) Tonic and phasic influence of basal forebrain unit activity on the cortical EEG. Behav Brain Res 115:159-170.

Detari L, Rasmusson DD, Semba K (1999) The role of the basal forebrain neurons in tonic and phasic activation of the cerebral cortex. Prog Neurobiol 58:249-277.

Everitt BJ, Robbins TW (1997) Central cholinergic systems and cognition. Annu Rev Psychol 48:649-684.

Fadel J, Moore H, Sarter M, Bruno JP (1996) Trans-synaptic stimulation of cortical acetylcholine release after partial 192 IgG-saporin-induced loss of cortical cholinergic afferents. J Neurosci 16:6592-6600.

Gill TM, Sarter M, Givens B (2000) Sustained visual attention performance-associated prefrontal neuronal activity evidence for cholinergic modulation. J Neurosci 20:4745-4757.

Greenwald AG, Gonzales R, Harris RJ, Guthrie D (1996) Effects sizes and p-values: what should be reported and what should be replicated. Psychophysiology 33:175-183.

Himmelheber A-M, Sarter M, Bruno JP (2000) Increases in cortical acetylcholine release during sustained attention performance in rats. Cogn Brain Res 9:313-325.

Keppel G (1991) Design and analysis, Ed 3. Englewood Cliffs, NJ: Prentice Hall.

Lehmann O, Grottick AJ, Cassel J-C, Higgins GA (2001) 192IgGSaporin-induced lesions of the basal forebrain: effects on attention as assessed by the 5-choice serial reaction time task. Behav Pharmacol 12[Suppl 1]:S58.

McGaughy J, Sarter M (1995) Behavioral vigilance in rats: task validation and effects of age, amphetamine and benzodiazepine receptor ligands. Psychopharmacology 117:340-357.

McGaughy J, Kaiser T, Sarter M (1996) Behavioral vigilance following infusions of $192 \mathrm{IgG}$-saporin into the basal forebrain: selectivity of the behavioral impairment and relation to cortical AChE-positive fiber density. Behav Neurosci 110:247-265.

McGaughy J, Sandstrom M, Ruland S, Bruno JP, Sarter M (1997) Lack of effects of lesions of the dorsal noradrenergic bundle on behavioral vigilance. Behav Neurosci 111:646-652.

Mesulam M, Mufson EJ, Levey AI, Wainer BH (1983a) Central cholinergic pathways in the rat: an overview based on alternative nomenclature (Ch1-Ch6). Neuroscience 10:1185-1201.

Mesulam M, Mufson EJ, Levey AI, Wainer BH (1983b) Cholinergic innervation of cortex by the basal forebrain: cytochemistry and cortical connections of the septal area, diagonal band nuclei, nucleus basalis (substantia innominata and hypothalamus) in the rhesus monkey. J Comp Neurol 214:170-197.

Metherate R, Ashe JH (1993) Nucleus basalis stimulation facilitates thalamocortical synaptic transmission in the rat auditory cortex. Synapse 14:132-143.

Metherate R, Weinberger NM (1990) Cholinergic modulation of responses to single tones produces tone-specific receptive field alterations in cat auditory cortex. Synapse 6:133-145.

Miner LA, Ostrander M, Sarter M (1997) Effects of ibotenic acid induced loss of neurons in the medial prefrontal cortex of rats on behavioral vigilance: evidence of executive dysfunction. J Psychopharmacology 11:169-178.

Muir JL, Everitt BJ, Robbins TW (1994) AMPA-induced excitotoxic lesions of the basal forebrain: a significant role for the cortical cholinergic system in attentional function. J Neurosci 14:2313-2326.
Muir JL, Everitt BJ, Robbins TW (1995) Reversal of visual attentional dysfunction following lesions of the cholinergic basal forebrain by physostigmine and nicotine and not by the 5-HT3 receptor antagonist, ondansetron. Psychopharmacology 118:82-92.

Muir JL, Everitt BJ, Robbins TW (1996a) The cerebral cortex of the rat and visual attentional function: dissociable effects of mediofrontal, cingulate, anterior dorsolateral and parietal cortex on a five choice serial reaction time task. Cereb Cortex 6:470-481

Muir JL, Bussey TJ, Everitt BJ, Robbins TW (1996b) Dissociable effects of AMPA-induced lesions of the vertical limb diagonal band of Broca on performance of the 5-choice serial reaction time task and on acquisition of a conditional visual discrimination. Behav Brain Res 82:31-44.

Parasuraman R, Giambra L (1991) Skill development in vigilance effects of event rate and age. Psychol Aging 6:155-169.

Parasuraman R, Warm JS, Dember WN (1987) Vigilance: taxonomy and utility. In: Ergonomics and human factors (Mark LS, Warm, JS, Huston, RL, eds), pp 11-32. New York: Springer.

Passetti F, Dalley JW, O'Connell MT, Everitt BJ, Robbins TW (2000) Increased acetylcholine release in the rat medial prefrontal cortex during performance of a visual attentional task. Eur $\mathbf{J}$ Neurosci 12:3051-3058.

Paxinos G, Watson C (1986) The rat brain in stereotaxic coordinates, Ed 2. Sydney: Academic.

Perry T-A, Hodges H, Gray JA (2001) Behavioral, histological, immunocytochemical consequences following 192 IgG-saporin immunolesions of the basal forebrain cholinergic system. Brain Res Bull 54:29-48.

Robbins TW, Everitt BJ (1995) Arousal systems and attention. In: The cognitive neurosciences (Gazziniga MS, ed), pp 703-720. Cambridge, MA: MIT.

Robbins TW, Everitt BJ, Marston HM, Wilkinson J, Jones GH, Page KJ (1989) Comparative effects of ibotenic acid and quisqualic acidinduced lesions of the substantia innominata on attentional function in the rat: further implications for the role of the cholinergic neurons of the nucleus basalis in cognitive processes. Behav Brain Res 35:221-240.

Stoehr JD, Mobley SL, Roice D, Brooks R, Baker LM, Wiley RG, Wenk GL (1997) The effects of selective cholinergic basal forebrain lesions and aging upon expectancy in the rat. Neurobiol Learn Mem 67:214-227.

Torres EM, Perry TA, Blokland A, Wilkinson LS, Wiley RG, Lappi DA, Dunnett SB (1994) Behavioural, histochemical and biochemical consequences of selective immunolesions in discrete regions of the basal forebrain cholinergic system. Neuroscience 63:95-122.

Turchi J, Sarter M (1997) Cortical acetylcholine and processing capacity: effects of cortical cholinergic deafferentation on cross-modal divided attention in rats. Cognit Brain Res 6:147-158.

Voytko ML (1996) Cognitive functions of the basal forebrain cholinergic system in monkeys: memory or attention? Behav Brain Res 75:13-25.

Voytko ML, Olton DS, Richardson RT, Gorman LK, Tobin JR, Price DL (1994) Basal forebrain lesions disrupt attention but not learning and memory. J Neurosci 14:167-186.

Waite JJ, Wardlow ML, Power AE (1999) Deficit in selective and divided attention associated with the cholinergic basal forebrain immunotoxic lesion produced by 192 IgG-saporin; motoric/sensory deficit associated with Purkinje cell immunotoxic lesion produced by OX7saporin. Neurobiol Learn Mem 71:325-352.

Wrenn CC, Lappi DA, Wiley RG (1999) Threshold relationship between lesion extent of the cholinergic basal forebrain in the rat and working memory impairment in the radial arm maze. Brain Res 847:284-298.

Zar JH (1974) Biostatistical analyses. Englewood Cliffs, NJ: Prentice Hall. 